\title{
THE DISTRIBUTION OF ELATINE MACROPODA GUSS. (ELATINACEAE)
}

\author{
AgNiEsZKa POPIELA ${ }^{1}$, ANDRZEJ ŁYSKO ${ }^{2}$ \\ ${ }^{1}$ Department of Botany and Nature Conservation, \\ University of Szczecin \\ Felczaka 3c, 71-412 Szczecin, Poland \\ e-mail:popiela@univ.szczecin.pl \\ ${ }^{2}$ Department of Environmental Protection and Management, \\ Western Pomeranian University of Technology \\ J. Słowackiego 17, 71-434 Szczecin, Poland
}

(Received: November 6, 2009. Accepted: January 6, 2010)

\begin{abstract}
Data on distribution of Elatine macropoda Guss. are given, listing distribution map and a list of localities. Disjunctive distribution pattern of the species within the Mediterranean region as well as distribution of other species of the section Elatinella Seub. and the genera suggests a long history of the range formation.
\end{abstract}

KEY WORDS: vascular plants, Elatinella, map, Isoëtalia, Mediterraneaen.

\section{INTRODUCTION}

Elatinaceae are a small cosmopolitan family of herbaceous aquatic and semi-aquatic plants and terrestrial shrubs composed of only just two genera, i.e. Elatine L., comprising about 15-25 taxa, and occurring in areas of moderate temperatures in both hemispheres, and Bergia L., with about 25 species occurring mostly in topical areas of the Old World, first of all in Africa and Australia (Tucker 1986; Leach 1989).

Both genera are very poorly known in respect of their taxonomy and phytogeography. No monograph of the genus Bergia has been published yet, whereas the only worldwide monograph of Elatine was published in the second half of the 19th century (Dumortier 1872). Of about 35-40 species of this family, barely a few have their general distribution mapped, i.e. E. hexandra, E. triandra, E. alsinastrum, and E. hydropiper (see Hultén 1971, Meusel et al. 1978, Hultén and Fries 1986, Lampe 1996). Subdivision of the genus Elatine into lower units was proposed by Niedenzu (1925, after Seubert 1845) who allocated - based on leaves arrangement on stem - the subgenus Potamopithys (Adanson) Seub. (whorled leaves) and the subgenus Elatine (Hydropiper Moesz.) Seub. (opposite leaves). The latter includes two sections: Elatinella Seub., where the number of stamens is double in relation to petals, and Triandra Seub. (=Crypta (Nutt. Seub.), characterised by the number of stamens being equal to that of petals (Tucker 1986). E. ma- cropoda is one of eight species of the section Elatinella, of which one is found in Euroasia, six in Europe (three of them also in North Africa) and one in North America (California).

The aim of this paper is to present data on the distribution of Elatine macropoda against a background of distribution of other species of the section Elatinella.

\section{METHODS}

The maps presented in this paper were made using the point-line method based on floristic and phytosociological literature, search query at the Kew Herbarium and the Herbarium of Natural History Museum in London, as well as the following web-based sources: Anthos - Information System of the Vascular Plants of Spain. Real Jardín Botánico, CSIC - Fundación Biodiversidad (www.anthos.es), consultations carried out in July 2009; biodiversity occurrence data provided by Herbario de la Universidad de Salamanca: SALA, Real Jardin Botanico (Madrid), Vascular Plant Herbarium (MA); Universidad de Extremadura, UNEX; Real Jardin Botanico (Madrid), Vascular Plant Herbarium (MA); Israel Nature and Parks Authority; Inventaire national du Patrimoine naturel (INPN) (Accessed through Data Portal of the Global Biodiversity Information Facility (GBIF), www.gbif.net, consultation carried out in July 2009. 


\section{RESULTS AND DISCUSSION}

Elatine macropoda is an annual herbaceous plant, about $10 \mathrm{~cm}$ long, with long internodes and most frequently upright stem and obovate or oblong obovate leaves, to $3 \mathrm{~mm}$ long. Quadruple flowers are placed on long pedicels. Eight stamens are found in two whorls, whereas seeds are slightly curved or comma-shaped, not very regularly punctate, about 21 rows of points.

Type: Sicily: In calcareis hyeme inundatis; Modica... (Gusson 1842). Most important synonimes: E. hydropiper subsp. campylosperma Seub. P. Fourn. (1936), E. hydropiper subsp. macropoda (Guss.) O. Bolos and Vigo (1990), E. campylosperma Seub., E. fabri Gren. (1838), E. hexandra subsp. macropoda (Guss.) Rouy and Foucaud in Rouy 1896.

Of eight species included in the section Elatinella Seub., Elatine macropoda is most probably the most closely related to European taxa, i.e. E. hydropiper, E. orthosperma, E. hungarica and E. gussonei, and to a North American species E. californica; they all are characterised by quadruple flowers, presence of eight stamens occurring in two whorls, but differ in pedicel length as well as seed shape and punctate pattern (Table 1). E. macropoda and E. gusso$n e i$ frequently are found in the erecta form, which is rare in other species - as a rule, they are procumbent and node-rooting plants. In the section Elatinella, also E. hexandra and $E$. brochonii with triple flowers are included but the relevance of distinguishing the section in such an approach still requires further studies.

Elatine macropoda is mainly found in the western and central part of the Mediterranean Basin, with the greatest number of its locations being known from the central part of the Iberian Peninsula while other are being scattered across France, the Italian Peninsula, northern shores of Africa, isles of the western and central part of the Mediter- ranean Sea and the Near East (Israel, Syria) (Fig. 1). E. macropoda is being found in flooded habitats in winter and early spring on communities of the alliance Isoëtion, most abundantly in the association Elatinetum macropodae Br.B1. (1931) 1935 or associations of the alliance Preslion. They occupy natural habitats, with human impact on them being only rarely observed, most frequently in the nearest surroundings of small water bodies (Moor 1936; Brullo and Minisale 1998)

In respect of the geographical range, Elatine macropoda lives next to E. gussonei - see Malta and Lampedusa (Brullo et al. 1988; Sommier 1908; Mifsud 2006); E. brochonii - single locations in the Atlantic part of south-western France (Clavaud 1883; Fiton 1916; Schotsman and Bosserdet 1966; Vanden Berghen 1966, Olivier et al. 1995), in the central part of the Iberian Peninsula (Cirujano and Velayos 1993), in Morocco (Hamada et al. 2004; Fennane and Ibn Tattou 2005), in the Mediterranean part of Algeria (Quézel and Santa 1962-1963) and in Corsica (Lorenzoni and Paradis 1997) and two E. hexandra - scattered locations, mainly in the southern part of the Central European Lowland, SW shores of the Scandinavian Peninsula, the Jutland Peninsula, W and SW British Isles, NW and Central France, and in addition in single locations on the Iberian Peninsula, in the northern Italy, Slovenia and Romania (Lampe 1996; Popiela (unpublished work)).

From among other species of the genus, the most widespread is E. triandra Schuhr., being found in all continents, except Antarctica. Some next species are reported from Asia and Australia (e.g. Albrecht 2002; Silvestre 1980; Prasad and Lal 1984), and about 10-15 species from the New World (Tucker 1986).

Disjunctive distribution pattern of Elatine macropoda within the Mediterranean region as well as distribution of other species of the section and the genera suggests a long

TABLE 1. Most important morphological traits of species of the section Elatinella Seub.

\begin{tabular}{|c|c|c|c|c|c|}
\hline Species & $\begin{array}{l}\text { Lenght of pedicel } \\
\text { (flower stalk) }\end{array}$ & $\begin{array}{l}\text { No } \\
\text { of sepals }\end{array}$ & $\begin{array}{l}\text { No } \\
\text { of petals }\end{array}$ & $\begin{array}{c}\text { No of } \\
\text { stamens }\end{array}$ & Seeds \\
\hline Elatine macropoda Guss. & long pedicel & 4 & 4 & 8 & $\begin{array}{l}\text { almost straight or curved, not very regular points, } \\
\text { about } 21 \text { rows of oblong points }\end{array}$ \\
\hline Elatine gussonei (Sommier) Brullo & long pedicel & 4 & 4 & 8 & $\begin{array}{l}\text { horseshoe-shaped or curved, about } 15 \text { rows of regular points, } \\
\text { hexagonal }\end{array}$ \\
\hline Elatine hungarica Moesz. & short pedicel & 4 & 4 & 8 & $\begin{array}{l}\text { horseshoe-shaped, or sickle-shaped: } 0.5 \mathrm{~mm} \text { long, } \\
\text { brownish }\end{array}$ \\
\hline Elatine hydropiper $\mathrm{L}$. & no pedicel & 4 & 4 & 8 & $\begin{array}{l}\text { horseshoe-shaped: brownish, } 0.3 \mathrm{~mm} \text { long, } \\
\text { transversely furrowed }\end{array}$ \\
\hline Elatine orthosperma Dueben & $\begin{array}{l}\text { no pedicel } \\
\text { or very short pedicel }\end{array}$ & 4 & 4 & 8 & $\begin{array}{l}\text { almost straight or slightly curved, } \\
\text { yellowish to brownish }\end{array}$ \\
\hline Elatine californica Gray & very short pedicel & 4 & 4 & 8 & $\begin{array}{l}\text { horseshoe-shaped, about } 15 \text { points per row, } \\
\text { as broad as long or broader than longer }\end{array}$ \\
\hline Elatine hexandra (Lapierre) DC & short pedicel & 3 & 3 & $6-8$ & $\begin{array}{l}\text { almost straight, fairly robust, about } 0.5 \mathrm{~mm} \text { long, } \\
\text { yellow-brown, shiny }\end{array}$ \\
\hline Elatine brochonii Clavaud & $\begin{array}{l}\text { nearly no pedicel } \\
\text { or very short pedicel }\end{array}$ & 3 & 3 & 6 & $\begin{array}{l}\text { almost straight, small (to } 0.3 \mathrm{~mm} \text { ), } \\
\text { reticulated, shiny }\end{array}$ \\
\hline
\end{tabular}




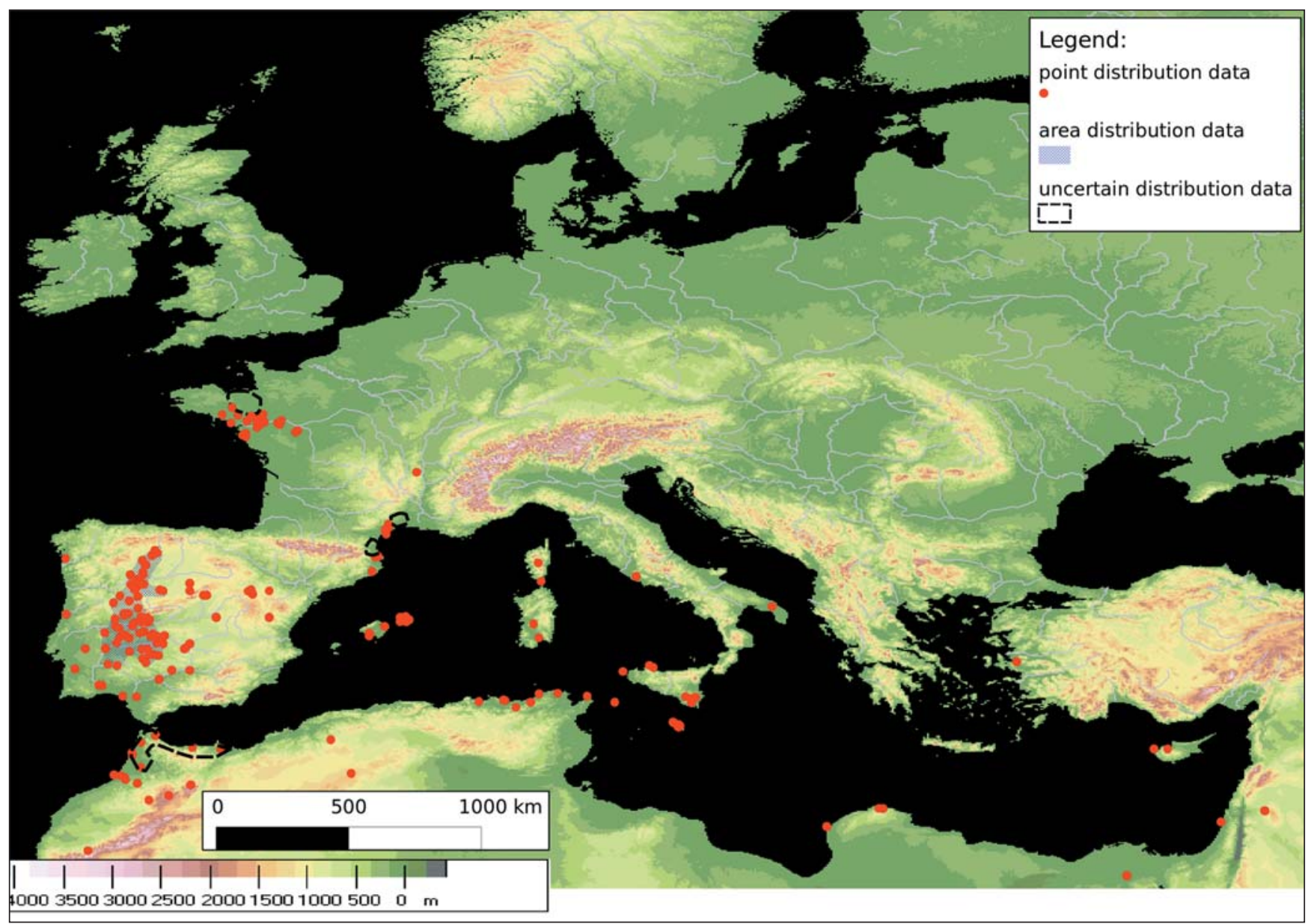

Fig. 1. The distribution of Elatine macropoda Guss. (Elatinaceae).

history of the range formation. Molecular phylogenetic studies, consisting in the comparison of sequences $r b c L, n d h F$ and $P H Y C$, have supported the hypothesis of Cronquist (1988), referring to a close relation between Elatinaceae and Clusiaceae and encompassing these two families within the order Malphigiales, with the closest taxonomic relations being seen between Elatinaeae and Malpigiaceae (Davis and Chase 2004; Heywood et al. 2007). A common ancestor of both families could have occurred in the Cretaceous period at the beginning of Albian, i.e. 111-100 million years ago, while both families separated presumably at the end of this period, i.e. about 98-89 million years ago (Davis and Chase 2004; Davis et al. 2005). Thus, it is possible to assume that history of the genus Elatine in the northern hemisphere can go down to the Mediterranean Tertiary and the Madrean Tertiary floras. Based on fossil data and molecular study results, Davis et al. (2001) have raised a hypothesis that the family Malphigiaceae originates in the northern part of South America and then their representatives migrated to North America and later, via northern Atlantic, to the Old World during certain Eocene episodes when climate favoured the development of tropical forests. The authors also suggest that the migration route proposed could explain many other disjunctive distribution patterns within the order Malphigiales.

\section{LIST OF LOCALITIES}

Algeria: Senhadia, Philippenville near Bone leg. Letourneux et Perraudiere, 1861(K); Tiaret, Aflou (Quezel and Santa 1963); near Bone, leg. ?, 1833 (K); near Bone, leg. Decaisne ? 1833, K; Oiseaux Lake between Bone and la Calle, leg. Gauthier-Lievre, 1925 (NHM).

Cyprus: Erymithes near Ayios Yeoryios (Akamas), leg. Meikle 1962 (K); between Baths of Aphrodite and Neokhorio, leg. Meikle, 1962 (K).

Egypt: El-Khanka near Cairo, 1880 (Bolos 2000).

France: Bretagne: Etang du Duc near Vannes, Etang de Duc, Etang de Millet near Ploërmel (Lloyd and Foucaud 1886). Ille-et-Vilaine (Coste 1937), Ferridon near Redon (Diard 2005). Pays-de-la-Loire: Luçon, Challans, St. Urbain, Bois de Céné, Thouaré, Vertou, lac de Grand-Lieu, Marsac, Guérande (Lloyd and Foucaud 1886). Maine-etLoire, Loire, Loire-Inferiere (Loir-Atlantique) (Coste 1937); Maine-et-Loir: Champtoce-su-Loir, leg. Willmott E. A. (K); Angers, leg. Moresce, 1899, (K); Foret d'Ancenis, etang de la Poiteviniere, foret de Saint-Mars-la-Jaille, foret et etangs de Vioreau (Corillon 1981, GBIF), La ChapelleBasse-Mer, near Oudon and Ancenis, Champtocé (Corillon 1981); Indre-et-Loire: Maille (GBIF).

\section{Abbreviations:}

K - Kew Herbarium; NHM - Natural History Museum, London; GBIF Data Portal of the Global Biodiversity Information Facility (GBIF); A Anthos. Information System of the plants of Spain. 
Languedoc-Rousillion: Hérault: Agde, in the Rigaud "mare", leg. R. Neyra, 1877, (K); near Agatham (Adge), leg. Wunderly, 1937, (K); Nr. Montpellier, Adge, leg. Guy, 1857, (K); Rigaud by Agde (Moor 1937); between Roquehaute and Portiragnes (Moor 1937); Gard, Hérault, Pyrenees-Orientales (Coste 1937). Rhone-Alpes: Loire: near Montbrison, leg. Le Grand, 1867, (K). Montbrison (Loire) : près du château de Vaugirard : Etang des Colombons (GBIF). Corse: Mt. Renoso, leg. Forsyth-Major, 1917, K. Isle Cavallo (Cavaddu) (Gamisans and Jeanmonod 1993; Jeanmonod and Gamisans 2007).

Spain: Badajoz (Cádiz), Cáceres, Córdoba, Ciudad Real, Gerona, Guadalajara, Huelva, León, Madrid, Islas Baleares [Maiorca, Menorca] Pontevedra, Salamanca, Sevilla, Segovia, Teruel, Toledo, Zamora (Cirujano and Velayos 1993). Majorka: Marina de Llucmajor (Barcelo 1977). Menorka: Binisarmeña (Barcelo and Combis 1880; Barcelo 1977). Badajoz: Campanario, río Zújar, Alange: Laguna Melchor Gomez, Azuaga: laguna el Hueco; Campanario: La Guarda. Río Ortigas a su paso bajo la carretera. de Quintana. Campillo de Llerena: Laguna del Guapero. Castuera: Arroyo Mejorada. Fregenal de la Sierra: Charca Los Leales. Helechosa de los Montes: Charca pista a Helechosa. La Roca de la Sierra: Laguna del Cortijo Tenerife. Magacela: El Berrocal. Puebla de Alcocer. Talarrubias. Valdecaballeros. Valencia de Mombuey. Villanueva del Fresno: Laguna Cuncos Norte (GBIF). Cáceres: Peraleda de la Mata, Navalmoral de la Mata. Ciudad Real: Villamayor de Calatrava, Laguna de Caracuel (Anthos: Information System of the plants of Spain). Alcalá de los Gazules. El Picacho, Alcalá de los Gazules. Sierra del Picacho, Chiclana de la frontera. Laguna de la Paja; Cc; Brozas, Cáceres, Dehesa de Clavín, Cáceres, Río Ayuela, Malpartida de Cáceres, finca el Majón, Malpartida de Plasencia, Dehesa de San Salvador, Navalmoral de la Mata, Peraleda de la Mata, Serrejón, Talayuela, Toril, Villa del Rey, Aceituna: Laguna Manzano. Aliseda: Charca Suertes de Godoy. Arroyo de la Luz: Laguna Grande. Cáceres: Charca Casas del Majón, Complejo Maruta, Complejo Maruta, Cañamero: Charca Estación Cañamero, Casas de Don Antonio, Guijo de Coria: Charca ctra Guijo de Coria, Ibahernando: Charca El Mago. Jaraíz de la Vera: Embalse de las Datas, Logrosán: Charca Badén de la Chaparra., Madroñera: Charca Ctra. Madroñera-Aldeacentenera, Monroy: Charca El Melonar. Monroy: Charca La Cierva de Arriba, Monroy: Laguna de Bremudo, Navalmoral de la Mata: Charca Arroyo Don Blasco, Charca Casa de la Chaparrera izquierda. Navas del Madroño: Charca de las Bardinas. Peraleda de la Mata, Serradilla: Embalse la Covachilla. Toril: Charca Macarra del Porquerizo Valencia de Alcántara: Charca Casas de la Ica. Villa del Campo: Charca del Sapo. Villa del Rey: Charca de Bramoso. Zarza la Mayor: Embalse de Benavente (GBIF).

Ciudad Real Almodóvar del Campo,Valle de Alcudia, Caracuel, Moral de Calatrava, Villamayor de Calatrava (GBIF). Córdoba: El Bramadero, Fuente Palmera, Belalcázar, río Guadamatilla, Alcolea Alcolea, río Guadamatilla, Santa Eufemia; Cardeña (Anthos: Information System of the plants of Spain; GBIF). Gerona: Alt Empordà, Pau, Rosas, Santa Cristina d'Aro, Baix Empordá, Calle di Roca Alba (Anthos: Information System of the plants of Spain; GBIF). Guadalajara: Campillo de Dueñas, El Cubillo de
Uceda, Fuentelahiguera de Albatages: Carramálaga, Nava de Ventas, La Yunta : La Torrecilla, Alto de Odón, de Alto de las Lagunillas, Torrecilla, los Llanos, Lagunas de Puebla de Beleña : Chica, Grande, Matarrubia, Tortuera, los Castellares, de Torrijo, las Cerradas, la Cruz del Pobre; Usanos, Valdenuño - Frenández, Arroyo de las Varguillas, Villaseca de Uceda, Viñuela (Anthos: Information System of the plants of Spain; GBIF). Huelva: H; El Rocio, Hinojos, marismas, Almonte. Reserva Biológica de Doñana, Laguna de las Pajas, Almonte. Reserva Biológica de Doñana, Laguna del Dulce, Coto de Doñana, Coto del Rey, Escacena, Hinojos, P.N. Doñana, Reserva de Doñana. En las márgenes del Lucio del Palacio, Sanlúcar de Guadiana, entre San Silvestre y Sanlúcar, Villanueva de los Castillejos, camino a Casa del Rioncillo (Anthos: Information System of the plants of Spain; GBIF). Balearic Islands: Mallorca: Marina de Llucmajor; Torre de Canyamel. Menorca: Binisarmenya, Ciutadella de Menorca, Son Toni Marti, Bassa des Mal Lloc, Es Mercadal, Albufera des Comte, Sa Bassa Verda, Es Migjorn Gran, Binicodrell de Baix, Ses Cuques (Anthos: Information System of the plants of Spain; GBIF). Madryt: Pinilla del Valle; Valdemanco. (Anthos: Information System of the plants of Spain; GBIF). León: Pozuelo del Páramo, Mansilla de las Mulas, Valdepolo (GBIF).

Salamanca Sa; Aldehuela de Yeltes, Babilafuente, El Cerro, Fuenteliante, Fuentes de Oñoro, La Fuente de San Esteban, Ledesma, Morasverdes, Rágama, laguna de Larajares, Aldehuela de Yeltes, Laguna del Cristo, Babilafuente, Espadaña, Becerril, Dos Cansas, Las Veguillas, Ledesma, Matilla de los Caños, Montemayor, Rágama (Anthos: Information System of the plants of Spain; GBIF). Sevilla: Écija, Laguna del Rodeo, Puebla del Rio,Dehesa Nueva (GBIF).

Segovia Cantalejo, Laguna de Navaelsoto, Cantalejo, Laguna de Navahornos, San Martín y Mudrián, Villarejo (Anthos: Information System of the plants of Spain; GBIF).

Teruel: Odón (GBIF). Toledo: Corchuela, Velada, Belvis de la Jara, Calera y Chozas, Talavera de la Reina (GBIF). Valladoid: Cervillejo de la Cruz, El Campillo, Carravillos, Tordesillas (GBIF). Zamora: Castropepe, Cubo de la Tierra del Vino, Fonfría, Manganeses de la Lampreana, Riego del Camino, Pereruela, Piedrahita de Castro, charca de Macias, Alfaraz, Castropepe, Peleas de Arriba, Peñausende, Pereruela, Piedrahíta de Castro, Macías, Riego del Camino, Salce (Anthos: Information System of the plants of Spain, GBIF). Zaragoza: Torralba de los Frailes, laguna del Montecillo (Anthos: Information System of the plants of Spain, GBIF).

Israel: Sharon Plain, Shefela, Lower and Upper Galilee, Golan (Danin 2004).

Libya: Benghazi, Shahat-Derna to Labrag; Roman aquaduct near Labrag, E of Shahat (Jafri El-Gadi 1980); Lamluda, Cyreneica, leg. R. Pampagnini et R. Pichi-Sermolli, 1934 (K); Lamluda, Derna Province, leg. N.J. Sandwith, N.D. Simpson, 1939 (NHM).

Malta: Balluta, leg. Mareh, 1872, (K); Island Malta, leg. J. F. Duthie, 1874, (K). St. Julians, leg. ? 1872 (K). Lampedusa: Isle Lampedusa, leg. Lojacono, 1888 (K); Wied Incita, Wied il-Ghasel, Wied Balluta, Minsija, Buskett, Talgholja; Gozo: Xlendi, Ta'Cenc, Munxar (Haslam et al. 1977).

Morocco: Takka-Ichiane, Ouked'meden, Moyen Atlas: near Kheneg Merzoul (Oued el Mansour), Region Médite- 
ranéenne (Fennane and Ibn Tattou 2005); W Rif; Loukkos, Cote Atlantique, Zerhoun, Marmara (Valdés et al. 2002); Tifflet, $5 \mathrm{~km}$ in SE, leg. Samuelsson 1936 (K); $22 \mathrm{~km} \mathrm{SW}$ of El Jadida, leg. M.R.K. Lambert, 1969 (NHM); Sale, Khemisset, Timhadit (Jahandiez and Maire 1932)

Portugal: Alto Alentejo, Baixo Alenteio, Beira Litoral (Cirujano and Velayos 1993). Elvas, Ajuda, Moinho do Benvindo, lezíria do Guadian (Malato-Beliz and Guerra 1977)

Syria: Djebel Druse. Bikes de la Foret de Qanaouat near El-Ayyoun (Mouterde 1970).

Tunesia: Guerah el-Akahl, leg. Cosson et al. 1883, K.; Kroumirie (Madjen el Ma), Mogods (Garaet Sedjenane), Cap Bon (Garaet el Haouaria) (Pottier-Alapetite 1951, 1979).

Turkey: Izmir (Davis 1967).

Italy: Italian Peninsula: paludi Pontine, Pen. Salentina, Favignana, Sicily (Fiori and Paoletti 1896-1898, Pignatti 1982); Sardinia: Cagliari, Monte Urpino, leg. P. Thomas, 1828, (K); Sardinia: Gesturi, leg. M. A. Garcia et al., 2003 (GBIF); Sicily: Palermo Mondello, Modica, Scisli, Spaccaforno, Siracusa, Ragusa, Noto (Gussone 1842); Island Favignata, leg. Huet du Pavillon, 1885, (K).

\section{ACKNOWLEDGEMENTS}

Funds were provided to A. Popiela by The Ministry of Science and Higher Education, Department of Scientific Research, grant No. PO4C03525. A. Popiela withes to thank the keepers of Kew Herbarium and of the Herbarium of Natural History Museum in London for access to herbarium material.

\section{LITERATURE CITED}

ALBRECHT D.E. 2002 Elatine macrocalyx (Elatinaceae), a new species from central and western Australia. Nuytsia 14. (3): 319-324.

BARCELÓ I., COMBIS F. 1880. Flora de las Islas Baleares. Imp. Pedro José Gelabert. Palma de Mallorca.

BARCELO C. 1977. Flora de Mallorca. 3. Editoral Moll Mallorca.

BOLOS L. 2000. Flora of Egipt. 2. Geraniaceae-Boraginaceae. Al Hadara Publishing, Cairo, p. 131.

BRULLO S., LANFRANCO E., PAVONE P., RONSISVALLE G. 1988. Taxonomical notes on the endemic flora of Malta. Nuovo Giorn. Bot. Ital., 122, Suppl. 1:9.

BRULLO S., MINISSALE P. 1998. Considerazioni sintassonomiche sulla classe Isoeto-Nanojuncetea. Itinera Geobot. 11: 263-290.

CIRUJANO S., VELAYOS M. 1993. Elatine L. In: S. Castroviejo (ed.), Flora Iberica, vol. 3. Real Jardín Botánico, CSIC, pp. 155-156.

CLAVAUD A. 1883. Communication. Actes Soc. Linn. Bordeaux 37: 52-55.

CORILLION R. 1981. Flore et végétation de la vallée de la Loire (cours occidental: de l'Orléanais l'estuaire). Jouve, Paris.

COSTE H. 1937. Flore descriptive et illustree de la France de la Corse et des contrées limitrophes. 1. Libraire scientifique et Technique, Paris.

CRONQUIST A. 1988. The Evolution and Classification of Flowering Plants second edition. The New York Botanical Garden, New York. 555 pp.

DANIN A. 2004. Distribution Atlas of Plants in the Flora Palestina Area. The Israel Academy of Sciences and Humanities.
DAVIS C.C., ANDERSON W.R., DONOGUE M.J. 2001. Phylogeny of Malpighiaceae: evidence from chloroplast $n d h \mathrm{~F}$ and trnL-F nucleotide sequences. Am. J. Bot. 88: 1830-1846.

DAVIS C.C., CHASE M.W. 2004. Elatinaceae are sister to Malpighiaceae; Peridiscaceae belong to Saxifragales. Am. J. Bot. 91: 262-273.

DAVIS C.C., WEBB C.O., WURDACK K.J., JARAMILLO C.A., DONOGHUE M.J. 2005. Explosive radiation of Malpighiales supports a mid-Cretaceous origin of modern tropical rain forests. Am. Nat. 165: E36-E65.

DAVIS P.H.F. 1967. Flora of Turkey and the Aegean Island. 2. Edinburg University Press.

DIARD L. 2005. Atlas de La Flore d'Ille-et-Vilane. Siloë edition, Nantes.

DUMORTIER B.C. 1872. Examen critique des Élatinacées. Bull. Soc. Roy. Bot. Belg. 11: 254-274.

FENNANE M., IBN TATTOU M. 2005. Flore vasculaire du Maroc, inventaire et chorologie. Vol. 1. Pteridophyta, Gymnospermae, Angiospermae (p.p.). Travaux de l'Institut Scientifique, Serie Botanique 37: 3-483.

FIORI A., PAOLETTI G. 1896-1898. Flora analitica d'Italia. Padova.

FITON J. 1916. Elatine brochonii Clavaud. Soc. Franç. Echange Pl. Bull. 6 : 28-30.

GAMISANS J., JEANMONOD D. 1993. Catalogue des plantes vasculaires de la Corse. In: Jeanmonod D., Burdel (éd.). Complements au Prodrome de la Flore de la Corse. Annexe nr 3. Conservatoire et Jardin botaniques Ville de Genève.

GUSSON G. 1842 Florć Siculć Synopsis. 1. Neapoli.

HAMMADA S., DAKKI M., IBN TATTOU M., OUYAHYA A., FENNANE M. 2004. Analyse de la biodiversité floristique des zones humides du Maroc. Flore rare, menacée et halophile. Acta Bot. Malacitana 29: 43-66.

HASLAM S.M., SELL P.D., WOLSELEY P.A. 1977. A Flora of the Maltese Islands. Malta University.

HEYWOOD V.H., BRUMMITT R.K., CULHAM A., SEBERG O. 2007: Flowering Plant Families of the World. Royal Botanic Gardens, Kew.

HULTÉN E. 1971. THE CIRCUMPOLAR PLANTS. 2. Dicotyledones. Kungl. Svenska Vetenskapsakademiens Handingar, Fjarde Serien 13(1): 1-463.

HULTÉN E., FRIES M. 1986. Atlas of North European vascular plants, north of the tropic of Cancer. 1. pp. XVIII + 498. Koelz Scientific Books, Königstein.

JEANMONOD D., GAMISANS J. 2007. Flora Corsica. Edisud, Aix-en Provence.

JAFRI S.M.H., EL-GADI A. (eds). 1980. Flora of Libya. 69-80. Al Faateh University, Department of Botany, Tripoli.

JAHANDIEZ E., MAIRE R. 1932. Cataloque des plantes du Maroc. 2. Imprimerie Minerva. Alger.

LAMPE VON M. 1996. Wuchsform, Wuchsrhytmus und Verbreitung der Arten der Zwergbinsengesellschaften. Diss. Bot. 266: $1-351$.

LEACH G.J. 1989. Taxonomic revision of Bergia (Elatinaceae) in Australia. J. Adelaide Bot. Gard. 11: 75-100.

LLOYD J., FOUCAUD J. 1886. Flore de l'ouest de la France. Nantes, Veloppé.

LORENZONI C., PARADIS G. 1997. Description phytosociologique d'une mare temporaire à Elatine brochonii dans le sud de la Corse. Bull. Soc. Bot. Centre-Ouest N. S. 28: 21-46.

MALATO-BELIZ J., GUERRA J.A. 1977. Notas de floristica X. Lagascalia 7(1) : 55-76.

MEUSEL H., JÄGER E., RAUSCHWERT S., WEINERT E. 1978. Vergleichende Chorologie der Zentraleuropaischen Flora. 2. Karten. ss. 259-421 G. Fischer Verlag, Jena. Press.

MIFSUD S. 2006. A comparative study between Elatine gussonei (from Malta) and Elatine macropoda (from Majorka). www. aquatic-gardeners.org/elatine.html (07.08.2009).

MOOR M. (1936): Zur Soziologie der Isoëtetalia. Beitr. Geobot. Landesaufn. Schweiz 20: 1-48. 
MOUTERDE P. 1970. Nouvelle Flore du Liban et de la Syrie. 2. Dar El-Macheq Editeurs. Beyrouth.

NIEDENZU F. 1925. Elatinaceae. In A. Engler and K. Prantl, Die natürlichen Pflanzenfamilien, 2nd ed., vol. 21, 270-276. W. Engelmann, Leipzig, Germany.

OLIVIER L., GALLAND J.P., MAURIN H., ROUX J.P. 1995. Livre Rouge de la flore menacée de France. Tome I: espèces prioritaires. Museum National d'Histoire Naturelle, Service Patrimoine Naturel, Conservatoire Botanique National de Porquerolles, Ministère de l'Environnement, Paris, 486 pages + annexes.

PIGNATTI S. 1982. Flora d'Italia. 3. Edagricole, p. 136-137.

POTTIER-ALAPETITE G. 1951. Note préliminaire sur l'Isoetion tunisien. Bull. Soc. Bot. France, 99: 4-6.

POTTIER-ALAPETITE G. 1979. Flore de la Tunisie. Angiospermes - Diotyledones. 1. Apetales Dialypetales. Imprimerie Officielle de la République Tunisienne.

PRASAD R. LAL J. 1984 Genus Elatine L. (Elatinaceae): a new record for Madhya Pradesh. J. Econ. Taxon. Bot., 5. (4): 981.

QUEZEL P., SANTA S., 1962-1963. Nouvelle flore de l'Algérie et des régions désertiques méridionales, 2 vol. Centre National Recherche Scientifique, Paris, 1170 pp.

SCHOTSMAN H.D., BOSSERDET P. 1966. Notes sur Elatine brochonii Clav. Bull. Cent. Etud. Rech. Sc. Biarritz 6(2): 251-267.

SEUBERT M. 1845. Elatinarum monographia. Acad. Caes. Leop. Nova Acta 21: 34-60.

SILVESTRE S. 1980 Notas breves: 13. Elatine brochonii Clavaud. Lagascalia 9. (2): 243-244.

SOMMIER S. 1908. Le isole Pelagie, Lampedusa, Linosa, Lampione e la loro flora, con un elenco delle piante di Pantelleria. Firenze Stab. Pellas, 345 pp.

TUCKER G.C. 1986. The genera of Elatinaceae in the southeastern United States. J. Arnold Arbor. 67: 471-483

VANDEN BERGHEN C. 1966. Deux localités nouvelles d'Elatine brochonii Clav. Bull. Cent. Etu. Rech. Sci. Biarritz 6(2): 269-270.

VALDÉS B., REJDALI M., ACHHAL EL KADMIRI A., JURY S.L., MONTSERRAT J.M. 2002. Caralogue de la plantes vasculaires du Nord du Maroc, incluant des Clés d'identification. Vol. 1. Universidad de Sevilla, Marid. 